\title{
Radiative decay of plasmons in a metallic nanoshell
}

\author{
T. V. Teperik* and V. V. Popov \\ Institute of Radio Engineering and Electronics (Saratov Division), Russian Academy of Sciences, Saratov 410019, Russia \\ F. J. García de Abajo \\ Centro Mixto CSIC-UPV/EHU and Donostia International Physics Center, Apartado Postal 1072, 20080 San Sebastian, Spain
}

(Received 10 August 2003; revised manuscript received 17 November 2003; published 2 April 2004)

\begin{abstract}
Retarded plasmon eigenmodes in metallic nanoshells are theoretically analyzed, and both plasmon eigenfrequencies and plasmon decay rates are calculated. Spherelike and voidlike plasmon modes are considered and their behavior with geometrical parameters is analyzed. Special attention is given to the problem of radiative decay of different plasmon modes supported by such systems. It is concluded that by varying the shell-layer thickness, the voidlike plasmon decay time can be varied over more than two orders of magnitude throughout the femtosecond range. For shell layers thinner than the characteristic skin depth, the voidlike plasmon modes exhibit subfemtosecond radiative lifetimes and hence they become more radiative than spherelike ones. For shell-layer thickness exceeding the characteristic skin depth, the decay time of the voidlike plasmons becomes of the order of tens of femtoseconds, yielding ultrahigh local-field enhancements. We predict local-field enhancement factors that exceed 60 and 150 in gold and silver nanoshells, respectively. These results are supported by calculations of absorption cross sections of these shells to external light. The results are applied to qualitatively explain strong coupling of plasmons with light in nanocellular metallic films, recently observed in light reflection experiments.
\end{abstract}

DOI: 10.1103/PhysRevB.69.155402

PACS number(s): 73.20.Mf, 61.46. $+\mathrm{w}, 78.47 .+\mathrm{p}$

\section{INTRODUCTION}

Electromagnetic eigenmodes of small metallic particles have attracted much attention in the past and are of great importance in current technology since they can be efficiently excited by light and their resonant frequencies can be tuned by varying the geometrical structure of metal nanoclasters and the dielectric properties of the host medium, or by choosing different metallic materials. ${ }^{1,2}$

Plasma oscillations excited in metallic particles cause a large field enhancement of the local field inside and near the particle. This filed enhancement is currently discussed for a great variety of potential applications. ${ }^{1,3,4}$ The local-field enhancement factor is given in the harmonic oscillator model ${ }^{2}$ by $|f|=\omega_{\text {res }} / 2 \gamma$, where $\omega_{\text {res }}$ is the resonance frequency and $2 \gamma$ is the homogeneous linewidth of resonance-the full width at half maximum (FWHM) of the resonance (see also Refs. 5 and 6). The linewidth of the resonance is related to the plasmon-energy decay time $\tau_{\mathrm{pl}}=1 / 2 \gamma$, where $\gamma$ is the plasmon-field decay rate. The value of $\tau_{\mathrm{pl}}$ can be measured using different nonlinear optical techniques ${ }^{6-11}$ or the spectral hole burning technique, ${ }^{12}$ or it can be extracted from the near-field spectra of the individual particles based on the near-field optical antenna effect. ${ }^{5}$ Ultrafast decay times on the sub-10-fs scale and local-field enhancement factors of up to 15 have been reported for circularly shaped metallic nanoparticles.

Recently, ${ }^{13}$ plasmon excitations in gold nanoporous films have been experimentally observed and their remarkable photonic properties established. It was presumed in Ref. 13 that plasmon modes excited in spherical nanocavities couple much more effectively to light than those in metallic spheres, which results in strong reflectivity resonances observed in experiment.
As an intuitive explanation of their observations, the authors of Ref. 13 developed a simple model of plasmon modes supported by a spherical void in an infinite metallic medium. Although that model gives the eigenfrequency values, which somehow can be fitted to the frequencies of resonances in the measured reflectivity spectra, it cannot describe the coupling between plasmon modes in the nanocavities and the radiation field. The reason is that the plasmon modes in a void are nonradiative because their electromagnetic field cannot radiate into the infinite metal having a negative dielectric function. At the same time, the enormous resonance peaks in the reflectivity spectra observed in Ref. 13 suggest strong coupling of nanocavity plasmons to the incident light. Therefore, an understanding of the effect of coupling between plasmons in metallic nanocavities and the radiation field becomes of great importance.

To examine the essential physics of coupling between light and plasmons in metallic nanocavities we analyze here a simple model of single metallic shell with a vacuum core, suspended in vacuum. The model of multilayered spherical cluster has been developed earlier ${ }^{14}$ on the basis of classical Mie theory ${ }^{15}$ and it is widely used in nano-optics ${ }^{1,16-22}$ mostly for calculation of stationary extinction spectra of nanocomposite metallic materials. Since metallic nanoshells display unique structurally tunable optical properties, ${ }^{17,22}$ the interest in the optical features of such complex particles grows steadily. However, the nonstationary process of radiative decay of plasmons in metallic nanoshell has not been addressed earlier neither theoretically nor experimentally.

In this paper we use the electromagnetic Mie theory in order to calculate the eigenfrequencies and decay rates of retarded plasmon modes in a metalic nanoshell. We specially focus on the problem of tunable radiative decay of plasmons supported by such a system, and show that the decay time of 

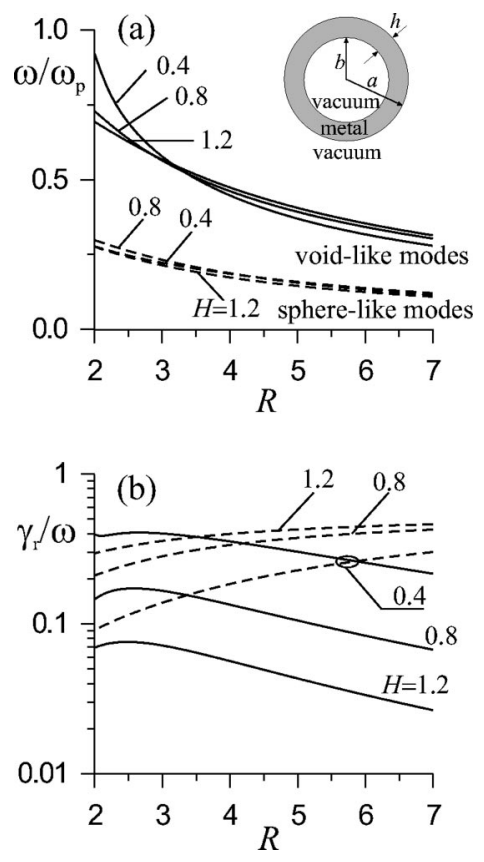

FIG. 1. (a), (b) Normalized eigenfrequencies $\omega / \omega_{p}$ and radiative decay rates $\gamma_{r} / \omega$ of the fundamental $(l=1)$ voidlike (solid curves) and spherelike (dashed curves) modes vs the normalized internal radius of the shell $R=b \omega_{p} / \mathrm{cl}$ for different values of normalized shell-layer thickness $H=h \omega_{p} / c l(l=1)$.

plasmons in metallic nanoshell can be effectively tuned over two orders of magnitude in the femtosecond domain. We estimate the local-field enhancement factor at plasmon resonances of a metallic nanoshell and show theoretically that for some nanoshell parameters the local-field enhancement factor in voidlike plasmon mode can reach ultrahigh values. It is shown by calculation of the absorption cross section of the shell to external light that such ultrahigh resonant enhancement of the local field produces sharply enhanced light absorption. We conclude that unique optical properties of metallic nanoshells pave the way towards various useful application of such particles in plasmon-resonance nanooptics.

\section{MODEL AND BASIC EQUATIONS}

Let us consider a metallic shell having external and internal radii $a$ and $b$, respectively (see the inset of Fig. 1). Suppose that inside the shell core and outside the shell is vacuum. We describe the dielectric response of the metal to an electric field $E \exp (-i \tilde{\omega} t)$ in the local Drude model as

$$
\varepsilon(\tilde{\omega})=1-\frac{\omega_{p}^{2}}{\tilde{\omega}\left(\tilde{\omega}+i \gamma_{e}\right)},
$$

where $\omega_{p}$ is the bulk plasmon frequency and $\gamma_{e}$ is the phenomenological bulk electron relaxation rate.

Being interested in exploring retarded plasmon eigenmodes supported by such a metallic shell we seek a solution of the Maxwell equations in each medium. We decompose the electromagnetic field in each medium into spherical har- monics, which, due to the spherical symmetry of the problem, become uncoupled and, therefore, can be considered independently. Since the field has to be finite in all points inside the shell core, we use the spherical Bessel function of the first kind of the $l$ th order, $j_{l}\left(k_{0} r\right)$, where $l$ is the orbital momentum quantum number, for describing the radial dependence of field of the $l$ th spherical harmonic inside the shell core. ${ }^{23}$ Here $k_{0}=\tilde{\omega} / c$ and $c$ is the speed of light. Inside the shell layer we use a combination of the spherical Hankel functions of the first and second kind, $h_{l}^{(1)}(k r)$ and $h_{l}^{(2)}(k r)$, respectively, where $k=\tilde{\omega} \sqrt{\varepsilon(\tilde{\omega})} / c$. With allowance for the scattering condition, which requires that only outgoing spherical waves exist at infinity $r \rightarrow \infty,{ }^{23}$ we seek a solution in the form of spherical Hankel functions $h_{l}^{(1)}\left(k_{0} r\right)$ in the host medium outside the shell.

Satisfying the conditions of continuity of tangential components of the electric and magnetic fields at internal and external surfaces of the shell, we obtain the eigenfrequency relation for electrical modes (those with zero radial component of the magnetic field ${ }^{23}$ ) supported by the metallic shell: ${ }^{24}$

$$
h_{l}^{(1)}\left(\rho_{a}\right) \alpha_{l}^{E}=\left[\rho_{a} h_{l}^{(1)}\left(\rho_{a}\right)\right]^{\prime} \beta_{l}^{E},
$$

where

$$
\begin{gathered}
\alpha_{l}^{E}=\varepsilon(\tilde{\omega})\left[\rho_{b} j_{l}\left(\rho_{b}\right)\right]^{\prime} A_{l}-j_{l}\left(\rho_{b}\right) B_{l}, \\
\beta_{l}^{E}=\varepsilon(\tilde{\omega})\left\{\varepsilon(\tilde{\omega})\left[\rho_{b} j_{l}\left(\rho_{b}\right)\right]^{\prime} C_{l}-j_{l}\left(\rho_{b}\right) D_{l}\right\}, \\
A_{l}=j_{l}\left(\eta_{b}\right)\left[\eta_{a} h_{l}^{(1)}\left(\eta_{a}\right)\right]^{\prime}-h_{l}^{(1)}\left(\eta_{b}\right)\left[\eta_{a} j_{l}\left(\eta_{a}\right)\right]^{\prime}, \\
B_{l}=\left[\eta_{b} j_{l}\left(\eta_{b}\right)\right]^{\prime}\left[\eta_{a} h_{l}^{(1)}\left(\eta_{a}\right)\right]^{\prime} \\
-\left[\eta_{b} h_{l}^{(1)}\left(\eta_{b}\right)\right]^{\prime}\left[\eta_{a} j_{l}\left(\eta_{a}\right)\right]^{\prime}, \\
C_{l}=j_{l}\left(\eta_{b}\right) h_{l}^{(1)}\left(\eta_{a}\right)-h_{l}^{(1)}\left(\eta_{b}\right) j_{l}\left(\eta_{a}\right), \\
D_{l}=\left[\eta_{b} j_{l}\left(\eta_{b}\right)\right]^{\prime} h_{l}^{(1)}\left(\eta_{a}\right)-\left[\eta_{b} h_{l}^{(1)}\left(\eta_{b}\right)\right]^{\prime} j_{l}\left(\eta_{a}\right),
\end{gathered}
$$

$\rho_{a}=a k_{0}, \rho_{b}=b k_{0}, \eta_{a}=a k, \eta_{b}=b k$, and the prime denotes differentiation with respect to the argument. The square root of $\varepsilon(\omega)$ is chosen here to have a non-negative imaginary part. Equation (2) essentially coincides with the corresponding relations for a coated sphere ${ }^{25}$ having a hollow core.

We treat the frequency $\tilde{\omega}$ in Eqs. (1) and (2) as a complex-valued quantity $\tilde{\omega}=\omega-i \gamma$. The real part of the complex-valued frequency gives the plasmon eigenfrequency while the imaginary part $\gamma$ is the total decay rate. It is obvious that $\gamma=\gamma_{r}$, the radiative decay rate, if we neglect the electron relaxation processes $\left[\gamma_{e}=0\right.$ in Eq. (1)]. Each $q$ th (in ascending order in the eigenfequency value) solution of Eq. (2) for a given orbital momentum quantum number $l$ yields the frequencies of electrical Mie modes $E_{l m q},{ }^{23}$ where $q$ is the radial quantum number. It must be noticed that Eq. (2) is independent of the azimuthal quantum number $m$ due to the spherical symmetry of the problem and, therefore, the eigenmodes are degenerate with respect to $m$. 
At large distance from the shell $r \gg a$ the Hankel function $h_{l}^{(1)}\left(k_{0} r\right) \quad$ can be approximated as $\exp \left\{i\left[k_{0} r-\pi(l\right.\right.$ $+1) / 2]\} / k_{0} r$ and the electromagnetic field away from the shell becomes proportional to $\exp \left[i k_{0}(r-c t)\right] / r$. Note that, for complex-valued frequency $\tilde{\omega}$, the wave vector $k_{0}$ has a complex value as well, $k_{0}=k_{0}^{\prime}-i k_{0}^{\prime \prime}$. The scattering conditions at $r \rightarrow \infty$ are satisfied by choosing a solution with $k_{0}^{\prime}>0$ corresponding to outgoing spherical waves at $r \gg a$. Such a solution with $k_{0}^{\prime}>0$ also has the property $k_{0}^{\prime \prime}>0$, meaning that the electromagnetic field grows in amplitude away from the metallic shell as $\propto \exp \left[k_{0}^{\prime \prime}(r-c t)\right] / r$, which describes the propagating amplitude front of the decaying mode. This amplitude-phase pattern of the electromagnetic field is typical of radiative eigenoscillations in open electrodynamic systems. ${ }^{23}$ Higher amplitudes of an electromagnetic field at points located farther away from the shell arise from radiation arriving at these points at a given instant of time due to radiative decay of plasmon oscillations in the shell at earlier instants of time. Similar time-space dependence of the field of radiatively decaying modes was established in various systems: e.g., radiative cyclotron-polaritons in a twodimensional electron plasma, ${ }^{26}$ leaky modes in photonic crystal-slab waveguide, ${ }^{27}$ and radiative exciton-polaritons in a quantum-well. ${ }^{28}$ Divergence of the solution for outgoing waves at $r \rightarrow \infty$, which is commonly referred to as exponential catastrophe, ${ }^{29}$ is discarded on the physical ground of considering the initial conditions. If we conceive the decaying process that begins at time $t=0$ then at a later time $t$ $>0$, the exponentially growing solution (with $r \rightarrow \infty$ ) has physical meaning only in a finite space domain $r<c t$ occupied by the decaying mode (see also relevant arguments in Refs. 27 and 28).

\section{RESULTS AND DISCUSSION}

To obtain the radiative decay rate of eigenmodes in the metallic shell, which is basically the parameter controlling the coupling between eigenoscillations and radiation field, ${ }^{30}$ we assume in our calculations $\gamma_{e}=0$ for a while. In this paper, only the electrical modes with $q=1$ will be considered. Only such plasmon modes can be excited in a shell with radius that is smaller than the light wavelength.

Figure 1(a) shows the calculated eigenfrequencies for fundamental plasmon Mie modes $E_{1 m 1}(l=1, q=1)$ in metallic shell versus the normalized internal radius of the shell $R$ $=b \omega_{p} / c l$ for different values of the normalized shell-layer thickness $H=h \omega_{p} / c l$, where $h=a-b$. It is seen in Fig. 1(a) that, for a metallic shell, each mode $E_{1 m 1}$ splits into two modes, voidlike $E_{1 m 1}^{(v)}$ and spherelike $E_{1 m 1}^{(s)}$ ones. The splitting originates from the coupling of plasmons bound to different (internal and external) surfaces of the shell. This doublet structure of optical spectra of metallic shells has been demonstrated theoretically,17,18 and observed experimentally. ${ }^{1,17}$ Similar effects occur in a planar metal film as well, where the resonance splitting is induced by the coupling of surface plasmon modes propagating at different boundaries of the film. ${ }^{31}$

As one can see in Fig. 1, the eigenfrequencies of voidlike
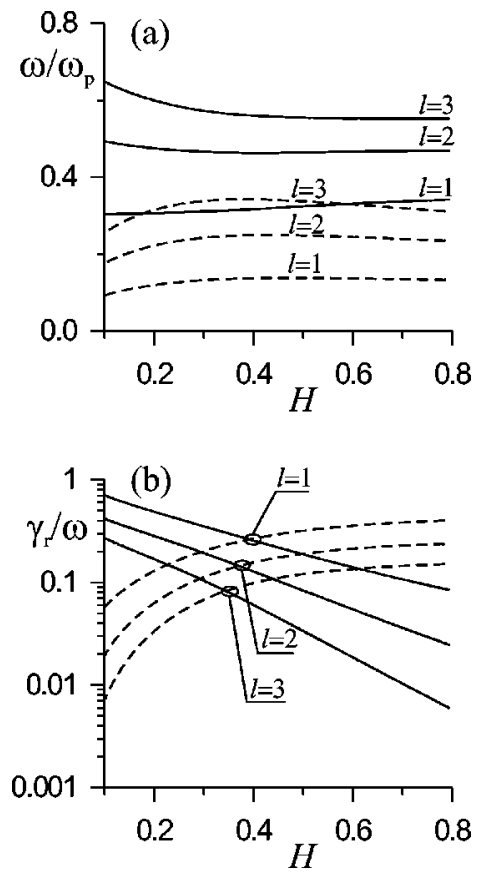

FIG. 2. (a), (b) Normalized eigenfrequencies $\omega / \omega_{p}$ and radiative decay rates $\gamma_{r} / \omega$ of different voidlike (solid curves) and spherelike (dashed curves) modes $E_{l m 1}^{(s, v)}(l=1,2,3)$ of a metallic shell vs the normalized shell-layer thickness $H=h \omega_{p} / c l$ for normalized internal radius $R=b \omega_{p} / c l=6$.

and spherelike plasmon modes do not change essentially with shell-layer thickness in the range of $H$ under consideration. However, the radiative decay rates of these modes change drastically with $H$ [cf. Figs. 1(a) and 1(b) and pay attention to the logarithmic scale over the ordinate axis, Fig. $1(\mathrm{~b})]$.

In Fig. 2, the eigenfrequencies and radiative decay rates are represented as a function of the normalized thickness of the shell layer for different plasmon modes $E_{l m 1}^{(v, s)}(l$ $=1,2,3$ ) and for $R=6$. For a gold shell $\left[\omega_{p}=7.9 \mathrm{eV}\right.$ (Ref. $32)$ ] this value of $R$ corresponds to an actual internal radius $b \simeq 150 \mathrm{~nm}$. It is seen in Fig. 2(b) that the radiative decay rate of the $l$ th mode decreases with increasing $l$. Although voidlike plasmons are bound to the internal surface of the shell, these modes can be more radiative than spherelike ones if the shell-layer thickness $h$ is small enough as compared with the characteristic screening length, $\delta_{l}=l \delta$, where $\delta=c / \omega_{p}$ is the characteristic skin depth $(\delta \simeq 25 \mathrm{~nm}$ for gold). At the normalized shell-layer thickness $H<0.4$, the fundamental voidlike mode exhibits the highest radiative decay rate. It is worth mentioning that one has $v_{l} / c<1$ for spherelike modes and $v_{l} / c>1$ for voidlike modes, where $v_{l}=b \omega / l$ is the orbital phase velocity. Such a fast orbital phase velocity peculiar to voidlike modes causes their enhanced radiative decay (see background arguments, e.g., in Ref. 23).

Figure 2(a) shows that the splitting between spherelike and voidlike modes increases with decreasing shell-layer thickness, starting with a normalized thickness $H \simeq 0.2$. The eigenfrequencies of spherelike and voidlike modes exhibit redshifts and blue shifts, respectively. This fact agrees with 

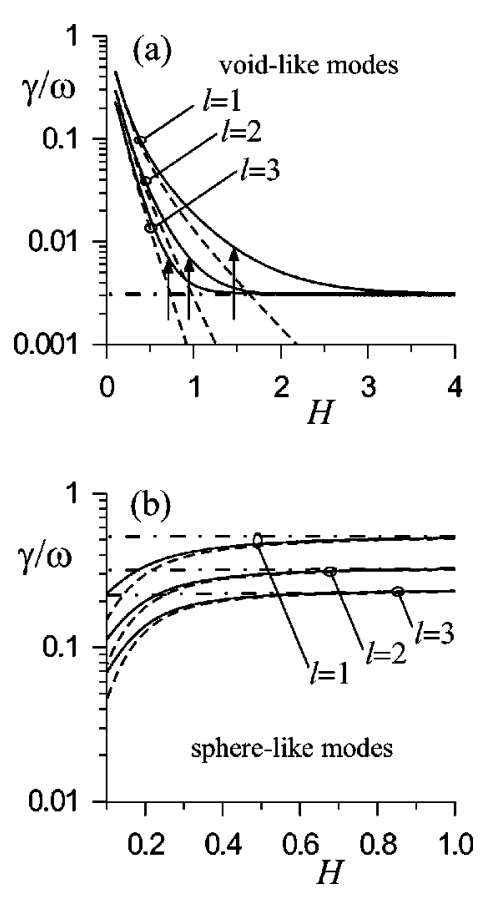

FIG. 3. Normalized total (solid lines) and radiative (dashed lines) decay rates of different (a) voidlike modes $E_{l m 1}^{(v)}$ and (b) spherelike modes $E_{l m 1}^{(s)}(l=1,2,3)$ of a gold shell vs the normalized shell-layer thickness $H=h \omega_{p} / \mathrm{cl}$ for normalized internal radius $R$ $=b \omega_{p} / c l=14$. The total decay rates of plasmons in a gold sphere of normalized radius $R^{\prime}=R+H$ in vacuum and the decay rates, $\gamma$ $=\gamma_{d}$, of plasmons in a void of normalized radius $R$ in infinite gold metal are shown by dash-dotted lines. Vertical arrows mark the points where $\gamma_{r}=\gamma_{d}$.

the blue shift (redshifts) of voidlike (spherelike) plasmon resonances in optical spectra of a metallic shell with decreasing shell-layer thickness as reported in Ref. 1.

To obtain the total decay rate $\gamma$, which includes both radiative and electron relaxation (dissipative) contributions, we chose the parameters $\omega_{p}=7.9 \mathrm{eV}$ and $\gamma_{e}=0.09 \mathrm{eV}$ (Ref. 32) in Eq. (1), which provide an optimum fit to gold bulk optical constants as reported in Ref. 33 within the investigated spectral region. In Fig. 3 the total decay rates of voidlike and spherelike plasmon modes are presented. The plasmon decay rates of a metallic sphere of radius $a$ in vacuum and of a void of radius $b$ in infinite metal are also shown by dash-dotted lines for comparison. Note that the decay rate of plasmons in the void is entirely of nonradiative origin and caused exclusively by electron relaxation processes. It is obvious that for $\gamma \ll \omega$ the difference between the total and radiative decay rates has to be attributed to the dissipative damping of plasmon modes due to electron relaxation processes $\gamma_{d}=\gamma$ $-\gamma_{r}$. Note that plasmon frequencies shift only slightly (less than $1 \%$ ), when an actual nonradiative damping is taken into account.

It is seen in Fig. 3(a) that for a small shell-layer thickness $h<\delta$ (i.e., $H<1$ ) the voidlike plasmons are overwhelmingly radiative, while they are predominantly dissipative for large thicknesses $h \gg \delta$. On the contrary, the electron relaxation contribution to the total decay rate of spherelike plasmons increases with decreasing shell-layer thickness down to $h$

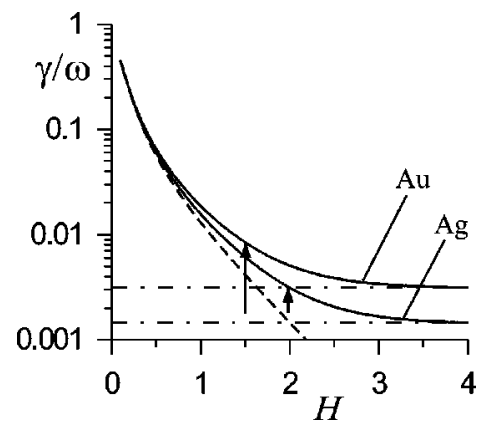

FIG. 4. Normalized total (solid lines) and radiative (dashed line) decay rates of the fundamental $(l=1)$ voidlike mode of gold and silver shells vs the normalized shell-layer thickness $H$ for $R=14$. The decay rates of plasmons in a void of normalized radius $R$ in infinite metal are shown by dash-dotted lines. Vertical arrows mark the points where $\gamma_{r}=\gamma_{d}$.

$<\delta$, while the spherelike plasmons are overwhelmingly radiative for shell-layer thickness $h \geqslant \delta$ [see Fig. 3(b)]. Therefore, the total decay rate $\gamma$ is controlled almost completely by ultrafast radiative processes with characteristic time in the range of a few femtoseconds for voidlike plasmons with $h$ $<\delta$ and for spherelike plasmons with $h \geqslant \delta$.

Figure 3 shows that the total decay rates of plasmons in metallic nanoshells vary dramatically over more than two orders of magnitude with varying shell-layer thickness $H$. Moreover, the decay rates of the voidlike plasmons are more sensitive to the shell-layer thickness than those of spherelike plasmons, and the range of variation of the total decay rate is larger for voidlike modes by one order of magnitude [see Figs. 3(a) and 3(b)]. Notice, however, that the total decay rate of plasmons in metallic spheres varies very little with radius $R^{\prime}$ [dash-dotted lines in Fig. 3(b)].

Now we can estimate the local-field enhancement factor at plasmon resonances as $|f|=\omega / 2 \gamma$. The value of $|f|$ is about 60 for the fundamental voidlike mode of gold shell with $H=1.5$ (the corresponding plasmon eigenfrequency is $1.39 \mathrm{eV})$. This value of $H$ corresponds to the case where $\gamma_{r}=\gamma_{d}$, i.e., $\gamma=2 \gamma_{r}$. We predict even larger local-field enhancement factors of up to 150 at $H=1.95$ (the corresponding plasmon eigenfrequency is $1.52 \mathrm{eV}$ ) for a silver shell, which are characterized by a smaller electron relaxation rate $\gamma_{e}=0.045 \mathrm{eV}$ and higher bulk plasmon frequency $\omega_{p}$ $=8.6 \mathrm{eV}$ (Ref. 34) (see Fig. 4). Note, for comparison, that the local-field enhancement factor obtained at plasmon resonance in a gold nanoball is below $15 .^{5,6}$ The total decay rate of the fundamental voidlike mode of metallic shells of different radii is shown in Fig. 5. One can see that the decay rate of voidlike plasmons at $h \gg \delta$ is smaller for metallic shells with larger core radius. Hence, the effect of local-field enhancement increases gradually with increasing $R$. It should be noted that, in the harmonic oscillator model, the relation $|f|=\omega / 2 \gamma$, where $\gamma$ is the plasmon decay rate, is equivalent to the relation $|f|=Q$, where $Q$ is the quality factor of the stationary plasmon resonance excited by incoming radiation. The local-field enhancement factor defined in such a way relates the amplitude of driven plasmon oscillations excited in the shell by incoming radiation at the resonance frequency 


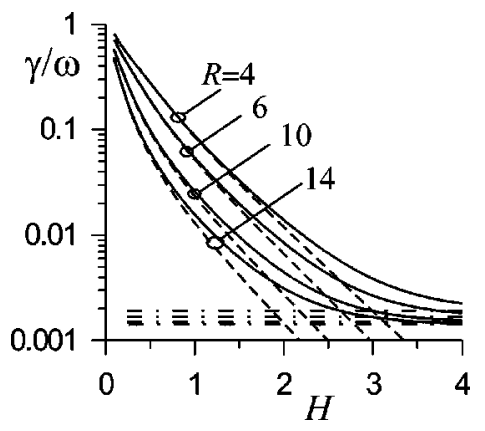

FIG. 5. Normalized total (solid lines) and radiative (dashed lines) decay rates of the fundamental $(l=1)$ voidlike mode of a silver shell vs the normalized shell-layer thickness $H$ at different normalized radii: $R=4,6,10$, and 14 , which correspond to actual internal radii $b \simeq 92.4,138.6,231$, and $323.4 \mathrm{~nm}$, respectively. The decay rates of plasmons in voids of normalized radius $R$ in infinite silver metal (dash-dotted lines) are presented for comparison.

to that far away from resonance frequency. However, it does not relate the amplitude of the local field inside or near the shell to the amplitude of incoming radiation (see also relevant arguments in Ref. 6).

We also have supported our estimates of the local-field enhancement factors by calculations of the excitation of stationary plasmon resonances in the shell by incoming light. The absorption cross section (see Fig. 6) has been obtained as the difference between the extinction and scattering cross sections calculated using the optical theorem and Mie coefficients corresponding to metallic shells., ${ }^{2,25}$ The parameters used in the calculation correspond to those marked by arrows in Fig. 4. The local-field enhancement factors as extracted from the absorption cross section spectra as the ratio between the resonance frequency and FWHM agree very well with those estimated above from the eigenmode theory within an error below 2\%. The height of the resonance peaks in Fig. 6 is measured from the smooth nonresonant background contribution. It should be noted that there is also an excellent agreement between the frequencies of the resonances shown in Fig. 6 and the corresponding frequencies of the plasmon eigenmodes, which are specified above, because the condition $\gamma \ll \omega$ is well satisfied in this particular case.

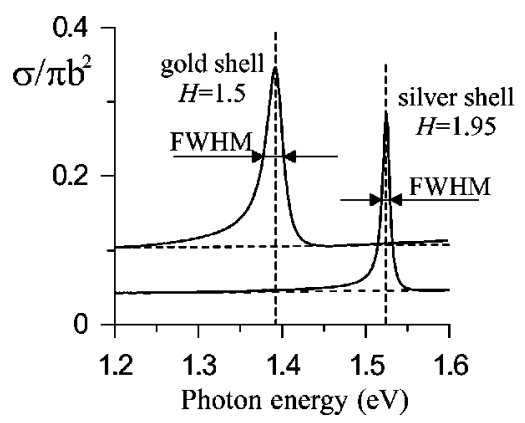

FIG. 6. Absorption cross section $\sigma$ for the voidlike plasmon resonance vs photon energy for gold and sliver shells with $R=14$ that are calculated for the shell parameters marked with arrows in Fig. 4 . The cross section has been normalized to the projected area of the shell core, $\pi b^{2}$.

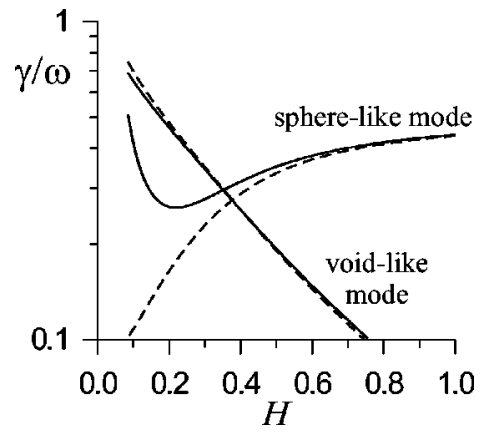

FIG. 7. Normalized total decay rates of the fundamental ( $l$ $=1$ ) voidlike and spherelike modes of a gold shell vs the normalized shell-layer thickness $H$ for $R=6$ with (solid lines) and without (dashed lines) surface electron scattering.

For a shell-layer thickness that is smaller than several nanometers, surface scattering of electrons should play a role in addition to bulk electron scattering. In this case, the overall electron relaxation rate can be estimated in the framework of the limitation of electron mean-free-path model, ${ }^{1,2}$ according to which the bulk electron relaxation rate $\gamma_{e}$ should be replaced by $\gamma_{e}+v_{F} / L$ in Eq. (1). Here $v_{F}$ is the electron velocity at the Fermi surface (which is of the order of $10^{8}$ $\mathrm{cm} / \mathrm{s}$ in metals) and $L$ is the effective mean-free path for electron collisions with the particle boundaries. In the approximation of diffuse scattering of electrons from the shell boundaries, ${ }^{18}$ one has

$$
L=a\left[\frac{1}{1+s^{2}}-\frac{s}{2}-\frac{1}{4} \frac{\left(1-s^{2}\right)}{\left(1+s^{2}\right)}(1-s) \ln \frac{(1-s)}{(1+s)}\right],
$$

where $s=b / a$.

Figure 7 shows that the surface electron scattering does not change appreciably the total decay rate of voidlike plasmons, whereas it drastically enhances the total decay rate of the spherelike plasmons at small values of $H$. As a result, the decrease in the radiative decay rate of spherelike plasmon modes for $H<1$ (i.e., $h<\delta$ ) is effectively canceled by the increase in surface scattering, so that the total decay rate of spherelike plasmons falls down only slightly and it exhibits a shallow dip at $H \simeq 0.2$, and then rises steeply with decreasing $H$. Notice the nontrivial behavior of the total decay rate of the voidlike plasmon mode at small values of $H$. Although the surface electron scattering opens an additional relaxation channel, the net total decay rate of the voidlike plasmons decreases slightly. To resolve this apparent paradox one must keep in mind that at large values of the decay rate $\gamma \simeq \omega$ radiative and dissipative decay processes are not independent of each other anymore. Then Fig. 7 suggests that the enhanced electron relaxation slows down the radiative decay of the voidlike plasmons at small values of $H$.

One can see from Fig. 6 that ultrahigh resonant enhancement of the local field, ultimately caused by the resonant excitation of voidlike plasmon oscillations, produces sharply enhanced light absorption. We suggest that enhanced absorption of similar origin results in sharp and deep extinction resonances in the reflectivity spectra observed in the experiments of Ref. 13 on nanoporous gold films. However, it 
should be noted that it is difficult to compare quantitatively the results of our calculations with experimental data of Ref. 13 because we are considering a different kind of objects (metallic nanoshells), which can only qualitatively model the actual experimental situation. More sophisticated theoretical analysis including multiple scattering of the light between different shells is underway, with a view to quantitatively explain the results of Ref. 13.

\section{CONCLUSIONS}

Radiatively decaying plasmon eigenmodes in a metallic nanoshell has been theoretically studied. The eigenfrequencies and decay rates of voidlike and spherelike plasmons have been calculated. It has been shown that, although voidlike plasmons are bound to the internal surface of the shell, these modes can be more radiative than spherelike ones for sufficiently thin shell layers as compared with the characteristic skin depth.

By varying the shell-layer thickness $h$, the plasmon decay time, $\tau_{\mathrm{pl}}=1 / 2 \gamma$, can be varied over two orders of magnitude in voidlike modes throughout the femtosecond range. Such decay times can be measured using one of well-developed time-resolved measurement techniques. ${ }^{5-12}$ The voidlike modes exhibit ultrashort radiative lifetimes of the order of 1 fs when $h$ is much less than the characteristic skin depth. For $h$ of the order of a few characteristic skin depths, the plasmon-energy decay time in the voidlike mode becomes of the order of tens of femtoseconds, which yields ultrahigh values of the local-field enhancement factor exceeding 60 and 150 for gold and silver nanoshells, respectively. The effect of local-field enhancement increases gradually with increasing the shell-core radius.

It is worth noting in this connection that, although the magnetic modes do not couple to plasma oscillations in spherical metallic particles, they should manifest themselves within the investigated spectral region if the shell-core radius grows to be of the order of the light wavelength. Hence these modes, as well as high-order $(q>1)$ electrical modes, can, in principle, show up in optical spectra of nanocellular metallic materials with cavities of large enough radius. These effects deserve further investigations. The interaction between light and plasmons in periodic ensembles of metallic nanoshells as well as in periodic ensembles of voids in metallic substrate also demands specific theoretical treatment.

We suggest that ultrahigh enhancement of the local-field in voidlike plasmon modes is responsible for deep extinction resonances in reflectivity spectra observed recently in nanoporous gold films. ${ }^{13}$ We conclude that a metallic nanoshell represents a unique object whose radiative and local-field properties can be effectively tuned over a wide range by nanoengineering the shell parameters. This makes such a nanoparticle very attractive for various applications in future submicron light technology.

\section{ACKNOWLEDGMENTS}

We thank S. V. Gaponenko, V. G. Golubev, and S. G. Tikhodeev for inspiring conversations. Helpful discussions with A. N. Ponyavina and O. Stengel are gratefully appreciated. This work was supported by the Russian Foundation for Basic Research (Grant No. 02-02-81031) and the Russian Academy of Science Program "Low-Dimensional Quantum Nanostructures." T.V.T. acknowledges the support from the President of Russia through the grant for young scientists MK-2314.2003.02, from the National Foundation for Promotion of Science, and from INTAS (Grant No. YSF 2002-153). F.J.G.A. acknowledges help and support from the University of the Basque Country UPV/EHU (Contract No. 00206.21513639/2001) and the Spanish Ministerio de Ciencia y Tecnología (Contract No. MAT2001-0946).
*Electronic address: teperik@ire.san.ru

${ }^{1}$ U. Kreibig and M. Vollmer, Optical Properties of Metal Clusters, Springer Series in Material Science Vol. 25 (Springer-Verlag, Berlin, 1995).

${ }^{2}$ C. Bohren and D. Hufmann, Absorption and Scattering of Light by Small Particles (Wiley, New York, 1998).

${ }^{3}$ S. Nie and S.R. Emory, Science 275, 1102 (1997).

${ }^{4}$ L. Novotny, R.X. Bian, and X.S. Xie, Phys. Rev. Lett. 79, 645 (1997).

${ }^{5}$ T. Klar, M. Perner, S. Grosse, G. von Plessen, W. Spirkl, and J. Feldmann, Phys. Rev. Lett. 80, 4249 (1998).

${ }^{6}$ B. Lamprecht, J.R. Krenn, A. Leitner, and F.R. Aussenegg, Phys. Rev. Lett. 83, 4421 (1999).

${ }^{7}$ K. Puech, F.Z. Henari, W.J. Blau, D. Duff, and G. Schmid, Chem. Phys. Lett. 247, 13 (1995).

${ }^{8}$ B. Lamprecht, A. Leitner, and F.R. Aussenegg, Appl. Phys. B: Lasers Opt. 64, 269 (1997).

${ }^{9}$ J.-H. Klein-Wiele, P. Simon, and H.-G. Rubahn, Phys. Rev. Lett. 80, 45 (1998).

${ }^{10}$ M. Simon, F. Träger, A. Assion, B. Lang, S. Voll, and G. Gerber, Chem. Phys. Lett. 296, 579 (1998).
${ }^{11}$ B. Lamprecht, A. Leitner, and F.R. Aussenegg, Appl. Phys. B: Lasers Opt. 68, 419 (1999).

${ }^{12}$ F. Stietz, J. Bosbach, T. Wenzel, T. Vartanyan, A. Goldmann, and F. Träger, Phys. Rev. Lett. 84, 5644 (2000).

${ }^{13}$ S. Coyle, M.C. Netti, J.J. Baumberg, M.A. Ghanem, P.R. Birkin, P.N. Bartlett, and D.M. Whittaker, Phys. Rev. Lett. 87, 176801 (2001).

${ }^{14}$ A.L. Aden and M. Kerker, J. Appl. Phys. 22, 1242 (1951).

${ }^{15}$ G. Mie, Ann. Phys. (Leipzig) 25, 377 (1908).

${ }^{16}$ J. Sinzig and M. Quinten, Appl. Phys. A: Solids Surf. 58, 157 (1994).

${ }^{17}$ S.J. Oldenburg, R.D. Averitt, S.L. Westcott, and N.J. Halas, Chem. Phys. Lett. 288, 243 (1998).

${ }^{18}$ S.M. Kachan and A.N. Ponyavina, J. Mol. Struct. 563-564, 267 (2001).

${ }^{19}$ E. Prodan, A. Lee, and P. Nordlander, Chem. Phys. Lett. 360, 325 (2002).

${ }^{20}$ E. Prodan, P. Nordlander, and N.J. Halas, Chem. Phys. Lett. 368, 94 (2003).

${ }^{21}$ E. Prodan and P. Nordlander, Nano Lett. 3, 543 (2003).

${ }^{22}$ E. Prodan, C. Radloff, N.J. Halas, and P. Nordlander, Science 302, 419 (2003). 
${ }^{23}$ L.A. Weinstein, Open Resonators and Open Waveguides (Golem, New York, 1969).

${ }^{24}$ The magnetic modes, i.e., those with zero radial component of the electric field, do not couple to plasma oscillations in spherical geometry and therefore are not considered here.

${ }^{25}$ F.J. García de Abajo, Phys. Rev. B 60, 6086 (1999).

${ }^{26}$ V.V. Popov, T.V. Teperik, and G.M. Tsymbalov, Pis'ma Zh. Eksp. Teor. Fiz. 68, 200 (1998) [JETP Lett. 68, 210 (1998)].

${ }^{27}$ S.G. Tikhodeev, A.L. Yablonskii, E.A. Muljarov, N.A. Gippius, and T. Ishihara, Phys. Rev. B 66, 045102 (2002).

${ }^{28}$ V.V. Popov, T.V. Teperik, N.J.M. Horing, and T.Yu. Bagaeva, Solid State Commun. 127, 589 (2003).
${ }^{29}$ H.M. Nussenzveig, Causality and Dispersion Relations (Academic Press, New York, 1972).

${ }^{30}$ V.V. Popov and T.V. Teperik, Pis'ma Zh. Tekh. Fiz. 27, No. 5, 42 (2001) [Tech. Phys. Lett. 27, 193 (2001)].

${ }^{31}$ H. Raether, Surface Plasmons, Springer Tracts in Modern Physics Vol. 111 (Springer, Berlin, 1988).

${ }^{32}$ M. Kreiter, S. Mittler, W. Knoll, and J.R. Sambles, Phys. Rev. B 65, 125415 (2002).

${ }^{33}$ D.W. Lynch and W.R. Hunter, Comments on the Optical Constants of Metals and an Introduction to the Data for Several Materials (Academic Press, Boston, 1985).

${ }^{34}$ I.R. Hooper and J.R. Sambles, Phys. Rev. B 65, 165432 (2002). 